\title{
Adiponectin Levels and Coronary Artery Disease among HIV-Infected Men
}

Louise Norton $^{1 *}$, Stephanie K Brodine ${ }^{1}$, Sheila Medina ${ }^{2,3}$, Hector Lemus ${ }^{1}$, Vincent Ricchiuti ${ }^{4}$, Gilbert Boswell ${ }^{5}$, Dylan Wessman ${ }^{6}$, David Krause ${ }^{6}$ and Nancy F Crum-Cianflone $e^{1,2,3}$

${ }^{1}$ Graduate School of Public Health, San Diego State University, San Diego, CA, USA

2Infectious Disease Clinic, Naval Medical Center San Diego, San Diego, CA, USA

${ }^{3}$ Infectious Disease Clinical Research Program, Uniformed Services University of the Health Sciences, Bethesda, MD, USA

${ }^{4}$ Diagnostic Laboratory Facility, Brigham and Women's Hospital and Harvard Medical School, USA

${ }^{5}$ Radiology Department, Naval Medical Center San Diego, San Diego, CA, USA

${ }^{6}$ Cardiology Clinic, Naval Medical Center San Diego, San Diego, CA, USA

\begin{abstract}
Objective: HIV-infected persons frequently experience lipodystrophy and are at higher risk of cardiovascular disease, but the impact of adipose-related hormones, such as adiponectin, on Coronary Artery Disease (CAD) remains unclear. We evaluated the association of plasma adiponectin levels and the presence of CAD.

Design: A cross-sectional study among HIV-infected men in a large HIV clinic.

Methods: HIV-infected participants underwent CT scan imaging to determine CAD as measured by Coronary Artery Calcium (CAC) scores categorized as no detectable disease (0), minimum disease (1-100), and significant disease $(>100)$. Factors associated with $C A D$, including adiponectin levels, were evaluated using multivariate logistic regression modeling.

Results: 213 HIV-infected men were evaluated with a median age of 43 years, median CD4 count of 583 cells/ $\mathrm{mm}^{3}$, and $69 \%$ had HIV RNA $<50$ copies/mL. Forty-nine percent had lipohypertrophy, $30 \%$ had hypertension, and $21 \%$ had a moderate/high Framingham risk score (FRS). Thirty-three percent $(n=71)$ had positive CAC scores. Seventy percent $(n=149)$ had a low adiponectin level $(<4.0 \mu \mathrm{g} / \mathrm{ml})$, including $66 \%$ with $C A C=0,76 \%$ with $C A C$ of $1-100$, and $81 \%$ with $C A C>100(p=0.09)$. Adiponectin levels were inversely correlated with lipohypertrophy, hypertension, and triglyceride levels $(p<0.05)$, while FRS was marginally correlated $\left(X^{2}=3.1, p=0.08\right)$. In the final multivariate model, increasing age (OR 3.8 per 10 year increase, $95 \% \mathrm{Cl} 2.56-5.71, p<0.001$ ) was significantly associated with CAC and adiponectin approached significance (OR 0.8 per $1 \mu \mathrm{g} / \mathrm{ml}$ increase, 95\% Cl 0.69-1.00, $\mathrm{p}=0.05$ ).

Conclusions: Low adiponectin levels are common among HIV-infected persons. Adiponectin is correlated with lipohypertrophy and lower levels approached significance with CAD. Understanding the role of adiponectin in the pathogenesis of CAD may allow for targeted treatment and prevention strategies among HIV-infected persons.
\end{abstract}

Keywords: HIV; Cardiovascular disease; Adiponectin; Coronary artery calcium scores; CAC

\section{Introduction}

Cardiovascular disease is a leading cause of morbidity and mortality among HIV-infected persons [1,2]. As HIV patients are living longer, they are increasingly developing cardiovascular and metabolic diseases, similar to the aging general population [1-4]. Additionally, some studies suggest that HIV patients are experiencing accelerated vascular aging $[5,6]$, although further data are needed. The exact risk factors for the higher risk of cardiovascular disease among HIV patients are unclear, but likely related to complex interactions between the virus itself, antiretroviral medications, increased immune activation, and metabolic changes [3,4,7-13].

Further information on the pathogenesis of cardiovascular disease among HIV-infected persons is needed. The identification of novel markers may not only enhance the understanding of underlying pathogenic mechanisms of cardiovascular disease, but also serve as indicators of underlying disease. Recently, inflammatory markers, such as high-sensitivity C-reactive protein (hsCRP), have emerged as indicators of and risk factors for cardiovascular disease [14-16]. Since HIV-infected persons have altered patterns of fat distribution, whether markers associated with fat deposition are related to cardiovascular disease is currently unclear.

Adiponectin is a plasma protein secreted exclusively from the adipose tissue into the bloodstream, and levels are inversely correlated with obesity $[17,18]$. The mechanism whereby obesity leads to low adiponectin levels is the result of increased oxidative stress on adipose tissue and down regulation of adiponectin receptors [19]. Further, in epidemiologic investigations, inverse associations have been reported between plasma adiponectin levels and the presence of type 2 diabetes mellitus, hypertension, and the metabolic syndrome [18,20]. Recently, adiponectin has attracted attention for its anti-atherogenic and antiinflammatory properties $[21,22]$. As such, hypoadiponectinemia has been associated with an increased risk of cardiovascular disease in the general population $[20,23,24]$. Little data, however, exists among HIV-infected persons with a single study associating low adiponectin levels with increased Framingham risk scores (FRS) [25]. Since HIV patients often have altered fat distribution patterns, which may result in lower adiponectin levels, and prior studies have linked lipodystrophy to cardiovascular disease [26-29], data on the association of plasma

*Corresponding author: Louise Norton, Graduate School of Public Health San Diego State University, 3976, 60th Street Unit 127, San Diego, CA 92115, USA, Tel: 619-985-4395; E-mail: louiseeliz@hotmail.com

Received April 01, 2013; Accepted April 29, 2013; Published May 09, 2013

Citation: Norton L, Brodine SK, Medina S, Lemus H, Ricchiuti V, et al. (2013) Adiponectin Levels and Coronary Artery Disease among HIV-Infected Men. J AIDS Clin Res 4: 208. doi:10.4172/2155-6113.1000208

Copyright: (C) 2013 Norton L, et al. This is an open-access article distributed under the terms of the Creative Commons Attribution License, which permits unrestricted use, distribution, and reproduction in any medium, provided the original author and source are credited. 
adiponectin levels and cardiovascular disease among HIV-infected persons are needed.

Understanding the potential role of plasma adiponectin in the pathogenesis of cardiovascular disease among HIV-infected persons may be important in the development of novel preventive and treatment strategies. Therefore, we conducted a study to evaluate the association of adiponectin levels and Coronary Artery Disease (CAD) as measured by Coronary Artery Calcium (CAC) scores among HIV-infected men. Additionally, we examined the association of adiponectin with Fatty Liver Disease (FLD), since it may share common risk factors to CAD.

\section{Methods}

\section{Study population}

We conducted a cross-sectional study to determine the factors associated with coronary atherosclerosis as detected by coronary artery calcium (CAC) [30] scores among HIV-infected men. The secondary objective examined the association between adiponectin levels and FLD as detected by Computed Tomography (CT) imaging. Our study population consisted of HIV-infected men ( $\geq 18$ years of age) receiving care at the Naval Medical Center San Diego (NMCSD); all participants were military active duty members, retirees, or dependents. Of the 223 participants in the original study [31],213 were included in the current analysis after excluding females $(\mathrm{n}=10)$. Only men were included in this study since the majority of the participants were male and adiponectin levels vary by gender [32]. All participants were confirmed HIVpositive by enzyme-linked immunosorbent assay and Western blot testing. Participants were enrolled between December 2008 and March 2010. The study was approved by the Institutional Review Boards at NMCSD and San Diego State University, and all participants provided written informed consent.

\section{Clinical data collection}

Study participants completed a questionnaire including data on demographics (age, ethnicity, and gender); history of tobacco, alcohol, and illicit drug use; and medical history. Research coordinators reviewed participants' medical records for data on medical conditions, medication use, and HIV-specific information including CD4 cell counts (nadir and current) by flow cytometry, HIV RNA level (Roche Amplicor, undetectable $<50$ copies $/ \mathrm{ml}$ ), and years of HIV seropositivity [defined as the time from HIV seroconversion (midpoint between last negative and first positive test) or first seropositivity to enrollment]. The CD4 cell count and plasma HIV RNA level were obtained from the laboratory records given that they were collected in close proximity to study enrollment. Information collected on antiretroviral medications included cumulative use of nucleoside/nucleotide reverse transcriptase inhibitors (NRTIs) and Protease Inhibitors (PIs). Current receipt of highly active antiretroviral therapy (HAART) was defined as the use of three or more antiretroviral drugs as per the guidelines [33]. Specific NRTIs (abacavir, tenofovir and didanosine) and PIs (ritonavir, atazanavir) were examined based upon the number of users as well as published studies showing that some antiretroviral medications may be associated with increased CAD risk [11-13].

Diabetes and hypertension were defined based on the participant's use of medications for these conditions, and excessive alcohol use was defined as $>140$ grams of ethanol/week [34]. The participant's 10-year cardiovascular risk was determined using the FRS [35]. Research coordinators measured the participants' weight and height for calculation of the Body Mass Index (BMI); overweight was defined as a BMI $25.0-29.9 \mathrm{~kg} / \mathrm{m}^{2}$, and obese as a BMI $\geq 30 \mathrm{~kg} / \mathrm{m}^{2}$.
Additionally, measurements of body circumferences (hip, thigh, and waist circumference, and waist/hip ratio) and skin-fold thickness (subscapular, suprailiac, biceps, and triceps using a standardized Lange skin-fold calipers) were obtained on the participant's right side, as previously described [36,37]. Three measurements of each body site were taken, and the mean measurement was utilized for each body site. Percent body fat was calculated by caliper measurements [36,38].

The participant's physician conducted a visual assessment to estimate fat gain or loss in six locations (cheeks, neck, breasts, abdomen, buttocks, and legs) since the time of HIV diagnosis. The physician's visual assessment of the participant's lipodystrophy was ranked on a scale of -3 to +3 , with negative scores indicating lipoatrophy, positive scores indicating lipohypertrophy, and 0 as no change [39].

\section{Laboratory data}

Laboratory data included fasting ( $\geq 10$ hours) glucose, total cholesterol, High-Density Lipoprotein (HDL), Low-Density Lipoprotein (LDL), and triglyceride levels. Additionally, blood was obtained for plasma adiponectin (multimeric enzyme-linked immunosorbent immunoassay, ALPCO Diagnostics, Salem, NH), hsCRP (lower limit of detection $<0.5 \mathrm{mg} / \mathrm{dl}$, Particle Enhanced Immunoturbidimetric Assay, Roche, Indianapolis IN), and Erythrocyte Sedimentation Rate (ESR, Modified Westergren Method). All tests were performed at the NMCSD clinical laboratory [certified by Clinical Laboratory Improvement Amendments (CLIA)], except the adiponectin assay which was performed at the Analytical Translational Core Laboratory at Brigham and Women's Hospital Laboratory. Adiponectin levels were stored and run at the same time to ensure calibration. A low adiponectin level was defined as $<4.0 \mu \mathrm{g} / \mathrm{ml}[40,41]$.

\section{CT imaging}

All participants underwent a Computerized Tomography (CT) scan, on the same day as laboratory testing, to determine the CAC score and assess for FLD. Participants underwent imaging using a single, multidetector CT scan (Siemens Definition Dual Source CT Scanner, Siemens Medical Solutions, Forsheim, Germany) without the administration contrast media. Prospectively gated axial $3 \mathrm{~mm}$ images were obtained at $120 \mathrm{kV}$ during a single breath hold. The scanning protocol captured images with a 330-milisecond gantry rotation time, an individual detector width of $0.6 \mathrm{~mm}$ with a reconstructed section width of $3 \mathrm{~mm}$, and temporal resolution of 165 milliseconds. CAC scores were performed on an Aquarius workstation (TeraRecon, San Mateo, California) and calculated as the sum of all lesions in each of the coronary arteries using Agatston units [42]. A CAC score of $>0$ was considered positive for detectable calcium, and a score of $>100$ was considered clinically significant. Limited $3 \mathrm{~mm}$ axial images were also obtained through the mid-liver, centered at the level of the portal vein, and spleen to estimate fat content [43]. FLD was defined as a liver-tospleen ratio of $<1.0$, using the mean values of three images taken in the right posterior, right anterior, and left lobe of the liver [44]. Images were processed on an Impax 6.3 workstation (AGFA).

\section{Statistical analysis}

The primary outcome of this study was to evaluate the association of adiponectin with the presence of CAD as defined by the CAC score categorized as no detectable disease (0), minimum detectable disease (1-100), and clinically significant detectable disease (>100). Our secondary outcome examined the relationship of adiponectin levels with FLD. Statistical analyses included descriptive statistics for all variables of interest. Frequency distributions of categorical variables 
are presented as numbers with percentages, and continuous variables as medians with interquartile ranges (IQR). Spearman correlation coefficients and chi-square tests were used to describe the correlations between adiponectin and continuous and categorical variables of interest, respectively. Univariate proportional odds logistic regression was used to determine the association of each variable with the threelevel CAC score (no detectable disease, minimum detectable disease, and clinically significant detectable disease), and then repeated with FLD as the secondary outcome.

Variables with a p-value $\leq 0.20$ in the univariate analyses were evaluated in the multivariate analysis. Multivariate analysis was conducted using ordinal logistic regression with forward stepwise addition of variables to derive the final model. The final regression model included all independent variables with a $\mathrm{p}$-value $<0.05$ or variables which were confounders (changed the odds ratio of adiponectin $>10 \%$ ). Collinearity among variables of interest was assessed using Spearman's correlation coefficient, chi-square, and Fisher's exact tests; variables that were highly correlated with other independent variables in the multivariate model were eliminated from the final model, except the most significant and clinically relevant variables. Separate final multivariate models for the outcomes of CAC score and FLD were created. In addition to the model for FLD as the outcome, an additional logistic regression analysis was performed for non-alcoholic fatty liver disease (NAFLD), by excluding participants with excessive alcohol intake. Odds ratios (OR) and adjusted odds ratios (AOR) with 95\% confidence intervals (CI) are presented. A p-value of $<0.05$ was considered statistically significant and all statistical analyses were performed using SAS version 9.2 (SAS Institute Inc., Cary, NC).

\section{Results}

\section{Baseline study population characteristics}

A total of 213 HIV-infected men were evaluated with a median age of 43 years (IQR 36-49); 51\% were Caucasian, 22\% African American, $14 \%$ Hispanic, and $13 \%$ other (Table 1 ). Thirty percent of participants had hypertension, $6 \%$ had diabetes, $2 \%$ were co-infected with hepatitis $\mathrm{C}$ virus (HCV), and $17 \%$ were current tobacco users. By FRS, $21 \%$ had a moderate/high risk score. The median BMI was $26.7 \mathrm{~kg} / \mathrm{m}^{2}$ (IQR 24-29); 98 (46\%) participants were overweight and 42 (20\%) obese. By physician's visual lipodystrophy assessment, 104 (49\%) of the

\begin{tabular}{|c|c|c|c|c|c|c|}
\hline Characteristic $^{a}$ & $\begin{array}{l}\text { Total Cohort } \\
\qquad N=213\end{array}$ & $\begin{array}{c}\text { Clinically Significant Disease } \\
(\text { CAC Score }>100) \\
N=16 / 213\end{array}$ & $\begin{array}{c}\text { Minimum Detectable Disease } \\
\text { CAC Score 1-100) } \\
\text { N=55/213 }\end{array}$ & $\begin{array}{c}\text { No Detectable } \\
\text { Disease }(\mathrm{CAC}=0) \\
\mathrm{N}=142 / 213\end{array}$ & OR $(95 \% \mathrm{Cl})$ & P-value \\
\hline \multicolumn{7}{|l|}{ Variable of Interest } \\
\hline Adiponectin, per $1.0 \mu \mathrm{g} / \mathrm{ml}$ increase & $3.1(2.2-4.4)$ & $2.5(2.0-3.7)$ & $2.8(2.1-3.9)$ & $3.2(2.3-4.4)$ & $0.8(0.7,1.0)$ & 0.06 \\
\hline \multicolumn{7}{|l|}{ Demographics } \\
\hline Age, per 10 years & $43(36-49)$ & $51(49-55)$ & $49(42-55)$ & $40(31-45)$ & $3.7(2.5,5.5)$ & $<0.0001$ \\
\hline Ethnicity & & & & & & 0.01 \\
\hline Black & $48(22.5 \%)$ & $1(2.1 \%)$ & $9(18.7 \%)$ & $38(79.2 \%)$ & $0.3(0.1,0.7)$ & \\
\hline Hispanic & $30(14.1 \%)$ & $2(6.7 \%)$ & $3(10.0 \%)$ & $25(83.3 \%)$ & $0.2(0.1,0.8)$ & \\
\hline Other & $27(12.7 \%)$ & $2(7.4 \%)$ & $8(29.6 \%)$ & $17(63 \%)$ & $0.7(0.3,1.8)$ & \\
\hline White & $108(50.7 \%)$ & $11(10.2 \%)$ & $35(32.4 \%)$ & $62(57.4 \%)$ & 1.0 & \\
\hline \multicolumn{7}{|l|}{ Clinical Information } \\
\hline \multicolumn{7}{|l|}{ Hypertension } \\
\hline No & $150(70.4 \%)$ & $9(6 \%)$ & $26(17.3 \%)$ & $115(76.7 \%)$ & 1.0 & \\
\hline Yes & $63(29.6 \%)$ & $7(11.1 \%)$ & $29(46.0 \%)$ & $27(42.9 \%)$ & $3.8(2.1,7.1)$ & $<0.0001$ \\
\hline Diabetes & & & & & & 0.001 \\
\hline No & $200(93.9 \%)$ & $13(6.5 \%)$ & $48(24.0 \%)$ & $139(69.5 \%)$ & 1.0 & \\
\hline Yes & $13(6.1 \%)$ & $3(23.0 \%)$ & 7 (53.9\%) & $3(23.1 \%)$ & $5.9(2.0,17.2)$ & \\
\hline CHD Risk Level by FRS & & & & & & $<0.0001$ \\
\hline Low & $169(79.3 \%)$ & $10(5.9 \%)$ & $34(20.1 \%)$ & $125(74.0 \%)$ & 1.0 & \\
\hline Moderate/High & $44(20.7 \%)$ & $6(14.0 \%)$ & $21(48.0 \%)$ & $17(38.0 \%)$ & $4.0(2.0,7.7)$ & \\
\hline Tobacco Use & & & & & & 0.50 \\
\hline Never & $106(49.8 \%)$ & $5(4.7 \%)$ & $28(26.4 \%)$ & $73(68.9 \%)$ & 1.0 & \\
\hline Past & $70(32.9 \%)$ & $7(10.0 \%)$ & $16(22.9 \%)$ & $47(67.1 \%)$ & $1.1(0.6,2.2)$ & \\
\hline Current & $37(17.3 \%)$ & $4(10.8 \%)$ & $11(29.7 \%)$ & $22(59.5 \%)$ & $1.5(0.7,3.3)$ & \\
\hline \multicolumn{7}{|l|}{ HIV-specific Information } \\
\hline Duration HIV infection (years) & $11(5-19)$ & $20(14-23)$ & $17(8-22)$ & $8(4-15)$ & $1.1(1.0,1.1)$ & $<0.0001$ \\
\hline CD4 count (cells $\left./ \mathrm{mm}^{3}\right)$,per 100 cells & $583(386-729)$ & $643(464-836)$ & $480(305-734)$ & $587(425-713)$ & $1.1(0.9,1.3)$ & 0.09 \\
\hline Nadir CD4 (cells/mm³), per 100 cells & $260(138-366)$ & $257(103-346)$ & $170(71-304)$ & $284(187-382)$ & $0.7(0.5,0.8)$ & 0.001 \\
\hline \multicolumn{7}{|l|}{ HIV RNA level (copies/ml) } \\
\hline$<50$ & $148(69.5 \%)$ & $13(8.8 \%)$ & $38(25.7 \%)$ & $97(65.5 \%)$ & 1.0 & \\
\hline$\geq 50$ & $65(30.5 \%)$ & $3(4.6 \%)$ & $17(26.2 \%)$ & $45(69.2 \%)$ & $0.8(0.4,1.5)$ & 0.50 \\
\hline \multicolumn{7}{|l|}{ Current HAART Use } \\
\hline No & $37(17.4 \%)$ & $1(2.7 \%)$ & $4(10.8 \%)$ & $32(86.5 \%)$ & 1.0 & \\
\hline Yes & $176(82.6 \%)$ & $15(8.5 \%)$ & $51(29.0 \%)$ & $110(62.5 \%)$ & $3.8(1.4,10.2)$ & 0.01 \\
\hline $\begin{array}{l}\text { Total months NRTI use, per } 12 \\
\text { months }\end{array}$ & $76(17.3-147)$ & $154.4(99-180.3)$ & $125(53-182)$ & $47(9.1-117.2))$ & $1.1(1.0,1.1)$ & $<0.0001$ \\
\hline Total months protease & $24.4(0-71)$ & $78.1(24.4-117)$ & $53(3.3-105)$ & $3.05(0-63)$ & $1.1(1.0,1.2)$ & 0.0001 \\
\hline
\end{tabular}


Citation: Norton L, Brodine SK, Medina S, Lemus H, Ricchiuti V, et al. (2013) Adiponectin Levels and Coronary Artery Disease among HIV-Infected Men. J AIDS Clin Res 4: 208. doi:10.4172/2155-6113.1000208

Page 4 of 9

\begin{tabular}{|c|c|c|c|c|c|c|}
\hline inhibitor use, per 12 months & & & & & & \\
\hline \multicolumn{7}{|l|}{ Abacavir } \\
\hline None & $139(65.3 \%)$ & $6(4.3 \%)$ & $29(20.9 \%)$ & $104(74.8 \%)$ & 1.0 & \\
\hline Past or Current Use & $74(34.7 \%)$ & $10(13.5 \%)$ & $26(35.1 \%)$ & $38(51.4 \%)$ & $2.9(1.6,5.1)$ & 0.0004 \\
\hline \multicolumn{7}{|l|}{ Didanosine } \\
\hline None & $153(71.8 \%)$ & $8(5.2 \%)$ & $33(21.6 \%)$ & $112(73.2 \%)$ & 1.0 & \\
\hline Past or Current Use & $60(28.2 \%)$ & $8(13.3 \%)$ & $22(36.7 \%)$ & $30(50.0 \%)$ & $2.7(1.5,5.0)$ & 0.001 \\
\hline \multicolumn{7}{|l|}{ Tenofovir } \\
\hline None & $61(28.6 \%)$ & $4(6.5 \%)$ & $9(14.8 \%)$ & $48(78.7 \%)$ & 1.0 & \\
\hline Past or Current Use & $152(71.4 \%)$ & $12(7.9 \%)$ & $46(30.3 \%)$ & $94(61.8 \%)$ & $2.1(1.1,4.3)$ & 0.03 \\
\hline \multicolumn{7}{|l|}{ Ritonavir } \\
\hline None & $109(51.2 \%)$ & $3(2.8 \%)$ & $19(17.4 \%)$ & $87(79.8 \%)$ & 1.0 & \\
\hline Past or Current Use & $104(48.8 \%)$ & $13(12.5 \%)$ & $36(34.6 \%)$ & $55(52.9 \%)$ & $3.6(1.2,6.6)$ & $<0.0001$ \\
\hline \multicolumn{7}{|l|}{ Atazanavir } \\
\hline None & $142(66.7 \%)$ & $8(6.0 \%)$ & $33(23.0 \%)$ & $101(71.0 \%)$ & 1.0 & \\
\hline Past or Current Use & $71(33.3 \%)$ & $8(11.3 \%)$ & $22(31.0 \%)$ & $41(57.7 \%)$ & $1.8(1.0,3.3)$ & 0.04 \\
\hline \multicolumn{7}{|l|}{ Laboratory Results } \\
\hline $\mathrm{ESR}$, per $10 \mathrm{~mm} / \mathrm{hr}$ & $10(7-18)$ & $11(9-24)$ & $13(10-19)$ & $10(7-18)$ & $1.1(0.9,1.4)$ & 0.12 \\
\hline hsCRP level (mg/dL) & $0.2(0.1-0.3)$ & $0.2(0.1-0.4)$ & $0.2(0.1-0.4)$ & $0.2(0.1-0.3)$ & $1.2(0.8,1.8)$ & 0.24 \\
\hline Total cholesterol, per $10 \mathrm{mg} / \mathrm{dL}$ & $183(158-211)$ & $170(161-220)$ & $192(164-217)$ & $182(157-204)$ & $1.0(0.9,1.1)$ & 0.18 \\
\hline $\mathrm{HDL}$, per $10 \mathrm{mg} / \mathrm{dL}$ & $40(35-49)$ & $39(33-46)$ & $41(35-46)$ & $40(34-50)$ & $0.9(0.7,1.1)$ & 0.37 \\
\hline LDL , per $10 \mathrm{mg} / \mathrm{dL}^{\mathrm{b}}$ & $102(86-129)$ & $102(70-129)$ & $109(92-136)$ & $98(83-129)$ & $1.0(0.9,1.1)$ & 0.22 \\
\hline Fasting glucose, per $10 \mathrm{mg} / \mathrm{dL}^{\mathrm{b}}$ & $92(86-99)$ & $99(89-109)$ & $93(87-103)$ & $91(85-97)$ & $1.2(1.0,1.4)$ & 0.01 \\
\hline Triglyceride level, per $100 \mathrm{mg} / \mathrm{dL}$ & $143(94-243)$ & $181(115-270)$ & $178(118-247)$ & $131(79-235)$ & $1.2(1.0,1.4)$ & 0.01 \\
\hline \multicolumn{7}{|l|}{ Weight and Fat Measurements } \\
\hline BMI $\left(\mathrm{kg} / \mathrm{m}^{2}\right)$ & $\begin{array}{c}26.7(24.2- \\
29.4)\end{array}$ & $27.2(25.4-29.4)$ & $25.1(24-28)$ & $27(24.3-30)$ & $1.0(0.9,1.1)$ & 0.15 \\
\hline Suprailiac measurement (mm) & $16(11-23.3)$ & $16.4(11-28)$ & $16(10-21)$ & $17(11.3-24)$ & $1.0(0.9,1.0)$ & 0.69 \\
\hline Subscapular measurement $(\mathrm{mm})$ & $21(16-27.3)$ & $20(17-27)$ & $22.3(14-29)$ & $21.1(16-26.3)$ & $1.0(0.9,1.0)$ & 0.66 \\
\hline Biceps measurement (mm) & $8(5-11)$ & $7.2(5-11.2)$ & $7(4-11)$ & $9(5-11)$ & $1.0(0.9,1.1)$ & 0.20 \\
\hline Triceps measurement (mm) & $16(11.3-21)$ & $18(10-22)$ & $15(9-19.3)$ & $17(13-21)$ & $0.9(0.9,1.0)$ & 0.24 \\
\hline Waist circumference $(\mathrm{cm})$ & $94(86-99)$ & $96(87-107)$ & $91(84-98)$ & 94 (86-99) & $1.0(1.0,1.0)$ & 0.31 \\
\hline Thigh circumference $(\mathrm{cm})$ & $56(52-60)$ & $53(51-58)$ & $54(50-57)$ & $57(53-61)$ & $0.9(0.9,1.0)$ & 0.01 \\
\hline Hip circumference $(\mathrm{cm})$ & $93(87-98)$ & $94(87-100.2)$ & $93(86-98)$ & $92.2(87-98)$ & $1.0,0.9,1.0)$ & 0.72 \\
\hline Waist/Hip ratio $(\mathrm{cm})$ & $1.0(0.9-1.0)$ & $1.0(1.0-1.1)$ & $1.0(0.9-1.0)$ & $1(0.9-1.0)$ & $2.2(0.3,15.6)$ & 0.41 \\
\hline Percent body fat ${ }^{b}$ & $26(23-30)$ & $29(25.3-33.5)$ & $27.1(23-31)$ & $26(23-29)$ & $1.0(1.0,1.1)$ & 0.02 \\
\hline \multicolumn{7}{|l|}{ Physician Visual Assessment } \\
\hline Lipohypertrophy & $104(48.8 \%)$ & $11(10.6 \%)$ & 37 (35.6\%) & $56(53.8 \%)$ & $3.1(1.7,5.6)$ & 0.0002 \\
\hline Lipoatrophy & $81(38.0 \%)$ & $11(13.6 \%)$ & $28(34.6 \%)$ & $42(51.8 \%)$ & $3.0(1.6,5.3)$ & 0.0002 \\
\hline
\end{tabular}

${ }^{a}$ Categorical variables expressed as number (percentage) and continuous variables as median (interquartile range)

${ }^{b}$ All values are $n=213$, except LDL $(n=200)$, glucose $(n=212)$ and percent body fat $(n=212)$ due to missing values

$\mathrm{BMI}$, body-mass index; CAC, coronary artery calcium score; CHD Risk level, coronary heart disease risk level; $\mathrm{Cl}$, 95\% confidence interval; ESR, erythrocyte sedimentation rate; FRS, Framingham Risk Score; HAART, highly active antiretroviral therapy; HDL, high-density lipoprotein; hsCRP, high sensitivity C-reactive protein; LDL, low-density lipoprotein; NRTI, nucleoside/nucleotide reverse transcriptase inhibitor; OR, odds ratio

Table 1: Study Population Characteristics and Univariate Associations with Coronary Artery Disease by CAC Score among 213 HIV-Infected Men.

participants had lipohypertrophy and 81 (38\%) had lipoatrophy; fiftyone patients (24\%) had both lipodystrophy and lipoatrophy.

Regarding HIV characteristics, the median duration of HIV infection was 11 years (IQR 5-19), the median current CD4 count was 583 cells $/ \mathrm{mm}^{3}$ (IQR 386-729), the median CD4 nadir was 260 cells/ $\mathrm{mm}^{3}$ (IQR 138-366), and 69\% had an undetectable HIV RNA level. One hundred seventy-six people (83\%) were current HAART recipients, the median duration of NRTI use was 76 months (IQR 17-147), and the median duration of protease inhibitor use was 24 months (IQR 0-71).

\section{Prevalence of coronary artery disease and fatty liver disease}

Thirty-three percent $(\mathrm{n}=71)$ of HIV-infected participants had a positive CAC score, of which $8 \%(\mathrm{n}=16)$ had a CAC $>100$ indicating clinically significant disease. The median age among those with a CAC score $>100$ was 51 years (IQR 49-55) and for a CAC score of 1-100 was 49 years (IQR 42-55). Thirteen percent (28/211) were diagnosed with FLD using CT imaging. Of those with FLD, 17 (59\%) had a CAC score $>0$. FLD was noted among $9 \%$ of HIV participants with a CAC score of $0,19 \%$ with a CAC of $0-100$, and $44 \%$ with a CAC $>100$. CAC and FLD were highly correlated $\left(X^{2}=11, \mathrm{p}<0.001\right)$.

\section{Adiponectin levels}

Among the study population, the median adiponectin level was 3.1 $\mu \mathrm{g} / \mathrm{ml}$ (IQR 2.2-4.4). Seventy percent $(\mathrm{n}=149)$ had a low adiponectin level $(<4.0 \mu \mathrm{g} / \mathrm{ml})$, including $66 \%$ with a $C A C=0,76 \%$ with a CAC of $1-100$, and $81 \%$ with a CAC $>100(\mathrm{p}=0.09)$. Regarding adiponectin 
levels and CAC, the median level was $3.2 \mu \mathrm{g} / \mathrm{ml}$ (IQR 2.3-4.4) for those with a CAC score of $0,2.8 \mu \mathrm{g} / \mathrm{ml}$ (IQR 2.1-3.9) for those with a CAC score of 1-100, and $2.5 \mu \mathrm{g} / \mathrm{ml}$ (IQR 2.0-3.7) for those with a CAC score $>100(\mathrm{p}=0.06)$. Median adiponectin level among those with FLD was $2.8 \mu \mathrm{g} / \mathrm{ml}$ (IQR 1.9-3.5) and for those without FLD was $3.1 \mu \mathrm{g} / \mathrm{ml}$ (IQR 2.3-4.4) $(\mathrm{p}=0.06)$.

Adiponectin levels were inversely correlated with hypertension, triglyceride levels, BMI, lipohypertrophy, duration of HIV infection, cumulative NRTI use, and use of didanosine (all with $\mathrm{p}<0.05$ ) (Table 2). Higher HDL levels were correlated with higher adiponectin levels. FRS approached significance with adiponectin level, and of those with moderate/high FRS, $33(75 \%)$ had a low adiponectin level $(<4.0 \mu \mathrm{g} / \mathrm{ml})$.

\section{Factors associated with coronary artery disease (CAD)}

Among HIV infected persons: In the univariate models, adiponectin had a marginally significant association with CAD by CAC score (OR 0.8 per $1 \mu \mathrm{g} / \mathrm{ml}, \mathrm{p}=0.06$ ). Other factors associated with CAD

CAD (OR 0.8 per $1.0 \mu \mathrm{g} / \mathrm{ml}, \mathrm{p}=0.05$ ). There were no significant associations with other variables including HIV-specific information, such as included increasing age, African American and Hispanic compared to Caucasian ethnicity, hypertension, diabetes, moderate/ high FRS, higher fasting glucose levels, higher triglyceride levels, greater percent body fat, lipohypertrophy, and lipoatrophy (Table 1). Regarding HIV-specific factors, CAD was associated with longer duration of HIV infection, lower nadir CD4 counts, current HAART use, cumulative NRTI use, and cumulative PI use. Individual antiretroviral drugs associated with CAD in the univariate models are shown in Table 1.

In the final multivariate analysis, older age (OR 3.8 per 10 years, $\mathrm{p}<0.0001)$ was significantly associated with the presence of CAD as detected by CAC scores. Low adiponectin levels were marginally

\begin{tabular}{|c|c|c|c|}
\hline Variable $^{a}$ & $\begin{array}{c}\text { Chi } \\
\text { Square }\left(\boldsymbol{X}^{2}\right)\end{array}$ & $\begin{array}{c}\text { Correlation } \\
\text { Coefficient }^{\mathrm{b}}(\boldsymbol{r})\end{array}$ & P-value \\
\hline \multicolumn{4}{|l|}{ Clinical Information } \\
\hline Hypertension & 7.6 & & 0.01 \\
\hline CHD Risk Level by FRS & 3.1 & & 0.08 \\
\hline \multicolumn{4}{|l|}{ HIV-specific Information } \\
\hline Duration HIV infection (years) & & -0.2 & 0.003 \\
\hline Total months NRTI use & & -0.2 & 0.001 \\
\hline Abacavir & 2.9 & & 0.09 \\
\hline Didanosine & 13.9 & & 0.0002 \\
\hline \multicolumn{4}{|l|}{ Laboratory Results } \\
\hline Triglyceride level (mg/dL) & & -0.3 & $<0.0001$ \\
\hline Fasting glucose (mg/dL) & & -0.1 & 0.05 \\
\hline hsCRP (mg/dL) & & -0.2 & 0.003 \\
\hline $\mathrm{HDL}(\mathrm{mg} / \mathrm{dL})$ & & 0.2 & 0.0005 \\
\hline \multicolumn{4}{|l|}{ Weight and Fat Measurements } \\
\hline BMI $\left(\mathrm{kg} / \mathrm{m}^{2}\right)$ & & -0.2 & 0.01 \\
\hline Waist circumference $(\mathrm{cm})$ & & -0.1 & 0.06 \\
\hline Waist/hip ratio $(\mathrm{cm})$ & & -0.1 & 0.06 \\
\hline Percent body fat & & -0.1 & 0.09 \\
\hline \multicolumn{4}{|l|}{ Physician Visual Assessment } \\
\hline Lipohypertrophy & 9.2 & & 0.002 \\
\hline
\end{tabular}

a Table shows only the variables significantly correlated with adiponectin. All variables were inversely correlated with adiponectin, except for HDL in which higher HDL levels were correlated with higher adiponectin levels

b Spearman correlation coefficient

Table 2: Factors Correlated with Low Plasma Adiponectin $(<4.0 \mu \mathrm{g} / \mathrm{ml})$ among HIV-infected Men associated with antiretroviral medications, in the final multivariate model (data not shown).

Factors associated with fatty liver disease (FLD): In the univariate analyses with FLD ( $\mathrm{n}=211$; two patients were excluded due to missing liver/spleen ratio data) as the outcome, FLD was inversely associated with HDL and adiponectin approached statistical significance (OR 0.7 per $1.0 \mu \mathrm{g} / \mathrm{ml}, \mathrm{p}=0.06)$. Higher levels of triglycerides, fasting glucose, waist circumference, BMI, percent body fat, and lipohypertrophy were positively associated with FLD (Table 3 ). In the final multivariate analysis, FLD was associated with elevated triglycerides (OR 1.3 per $100 \mathrm{mg} / \mathrm{dL}, \mathrm{p}=0.01$ ) and increased waist circumference (OR 1.1 per 1 $\mathrm{cm}, \mathrm{p}<0.0001)$. Low adiponectin levels were not significantly associated with FLD in the final multivariate model (OR 0.8 per $1.0 \mu \mathrm{g} / \mathrm{ml}, \mathrm{p}=0.33$ ) (data not shown).

Since FLD may be secondary to NAFLD or excessive alcohol use, we conducted additional analyses excluding those with excessive alcohol use $(n=12)$, and observed similar findings. In the final multivariate model, adiponectin was not significant (OR 0.8 per $1.0 \mu \mathrm{g} /$ $\mathrm{ml}, \mathrm{p}=0.18$ ), but elevated triglycerides (OR 1.3 per $100 \mathrm{mg} / \mathrm{dL}, \mathrm{p}=0.007$ ) and waist circumference (OR 1.1 per $1 \mathrm{~cm}, \mathrm{p}=0.0002)$ were significantly associated with NAFLD.

\section{Discussion}

To our knowledge, this is the first study to examine adiponectin levels among HIV-infected men and evaluate its association with CAD as determined by CAC scores. Our study found that low adiponectin levels are common (70\% prevalence) among HIV-infected men. We also demonstrated that CAD was marginally associated with low adiponectin levels, and noted a dose relationship between higher CAC scores and lower adiponectin levels. These data suggest that low adiponectin levels may play a role in the pathogenesis of cardiovascular disease among HIV-infected persons.

Adiponectin is a plasma protein secreted from adipose tissue with anti-inflammatory, anti-oxidative, and vasodilator effects. Obesity is associated with reduced plasma adiponectin levels, and rates of obesity are increasing among HIV-infected persons [45,46]. Further, HIV patients are predisposed to lipodystrophy, likely an effect of both HIV itself and antiretroviral agents [17,47]. Studies have shown that certain antiretroviral agents (e.g., NRTIs such as stavudine and PIs such as ritonavir) accumulate in adipocytes and down-regulate Peroxisome Proliferator-Activated Receptor-gamma (PPAR- $\gamma$ ) and adiponectin secretion [48-52]. Hence similar to hypoadiponectemia associated with obesity, dysfunctional adipose tissue in the setting of lipodystrophy and antiretroviral agents may lead to reduced secretion of adiponectin, with resultant adverse cardiovascular effects [53-57].

Regarding CAD, reduced circulating levels of adiponectin in the bloodstream contribute to endothelial vascular dysfunction $[18,21]$. Further, adiponectin prevents atherosclerosis by its anti-inflammatory and anti-oxidative activities in both cardiac and vascular cells, and by preventing the uptake of cholesterol $[19,55]$. In a case-control study of males in the general population who underwent coronary angiography, males with hypoadiponectinemia had a 2 -fold increase in CAD prevalence, independent of traditional CAD risk factors [40]. Although lower levels of adiponectin have been associated with vascular diseases in the general population $[40,58,59]$, there is a paucity of data regarding the relationship of adiponectin levels and CAD among HIV-infected persons.

Our study is novel as it's the first to evaluate the relationship 
Citation: Norton L, Brodine SK, Medina S, Lemus H, Ricchiuti V, et al. (2013) Adiponectin Levels and Coronary Artery Disease among HIV-Infected Men. J AIDS Clin Res 4: 208. doi:10.4172/2155-6113.1000208

Page 6 of 9

\begin{tabular}{|c|c|c|c|c|}
\hline \multirow{2}{*}{ 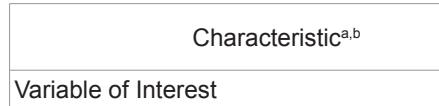 } & \multirow[t]{2}{*}{$\begin{array}{l}\text { Fatty Liver Disease } \\
\mathrm{N}=28(13.27 \%)\end{array}$} & \multirow[t]{2}{*}{$\begin{array}{c}\text { No Fatty Liver Disease } \\
183(86.73 \%)\end{array}$} & \multicolumn{2}{|c|}{$\begin{array}{l}\text { OR }(95 \% \mathrm{CI}) \\
\text { p-value }\end{array}$} \\
\hline & & & & \\
\hline Adiponectin, per $1.0 \mu \mathrm{g} / \mathrm{ml}$ increase & $2.8(1.9-3.5)$ & $3.1(2.3-4.4)$ & $0.7(0.5,1.0)$ & 0.06 \\
\hline \multicolumn{5}{|l|}{ Demographics } \\
\hline Age, per 10 years & $45(40-50)$ & $43(34-50)$ & $1.3(0.8,1.9)$ & 0.24 \\
\hline Ethnicity & & & & 0.20 \\
\hline Black & $2(4.3 \%)$ & $45(95.7 \%)$ & $0.2(0.04,0.9)$ & \\
\hline Hispanic & $4(13.3 \%)$ & $26(86.7 \%)$ & $0.7(0.2,2.3)$ & \\
\hline Other & $3(11.1 \%)$ & $24(88.9 \%)$ & $0.6(0.1,2.1)$ & \\
\hline White & $19(17.8 \%)$ & $88(82.2 \%)$ & 1.0 & \\
\hline \multicolumn{5}{|l|}{ Clinical Information } \\
\hline \multicolumn{5}{|l|}{ Hypertension } \\
\hline No & $17(11.4 \%)$ & $132(88.6 \%)$ & 1.0 & \\
\hline Yes & $11(17.7 \%)$ & $51(82.3 \%)$ & $1.7(0.7,3.8)$ & 0.22 \\
\hline \multicolumn{5}{|l|}{ Diabetes } \\
\hline No & $25(12.6 \%)$ & $173(87.4 \%)$ & 1.0 & \\
\hline Yes & $3(23.1 \%)$ & $10(76.9 \%)$ & $2.1(0.5,8.0)$ & 0.29 \\
\hline Tobacco Use & & & & 0.48 \\
\hline Never & $12(11.4 \%)$ & $93(88.6 \%)$ & 1.0 & \\
\hline Past & $9(12.9 \%)$ & $61(87.1 \%)$ & $1.1(0.4,2.9)$ & \\
\hline Current & $7(19.4 \%)$ & $29(80.6 \%)$ & $1.9(0.7,5.2)$ & \\
\hline \multicolumn{5}{|l|}{ HIV-specific Information } \\
\hline Duration HIV infection (years) & $11(6-22)$ & $11(5-19)$ & $1.0(0.9,1.0)$ & 0.61 \\
\hline CD4 count (cells $/ \mathrm{mm}^{3}$ ), per 100 cells & $601.5(394-766)$ & $578(384-713)$ & $1.0(0.9,1.2)$ & 0.56 \\
\hline Nadir CD4 (cells $/ \mathrm{mm}^{3}$ ), per 100 cells & $252.50(95-368)$ & $260(144-356)$ & $0.9(0.7,1.2)$ & 0.73 \\
\hline \multicolumn{5}{|l|}{ HIV RNA level (copies/ml) } \\
\hline$<50$ & $19(13.0 \%)$ & $127(87.0 \%)$ & 1.0 & \\
\hline$\geq 50$ & $9(13.9 \%)$ & $56(86.1 \%)$ & $1.1(0.4,2.5)$ & 0.87 \\
\hline \multicolumn{5}{|l|}{ Current HAART Use } \\
\hline No & $5(13.5 \%)$ & $32(86.5 \%)$ & 1.0 & \\
\hline Yes & $23(13.2 \%)$ & $151(86.8 \%)$ & $1.0(0.3,2.8)$ & 0.96 \\
\hline Total months NRTI use, per 12 months & $89.1(17-157.1)$ & $74.3(17.3-145.4)$ & $1.0(0.9,1.1)$ & 0.68 \\
\hline Total months protease & $36(0-78.1)$ & $23.40(0-71)$ & $1.0(0.9,1.1)$ & 0.33 \\
\hline \multicolumn{5}{|l|}{ inhibitor use, per 12 months } \\
\hline \multicolumn{5}{|l|}{ Ritonavir } \\
\hline None & $10(9.3 \%)$ & $97(90.7 \%)$ & 1.0 & \\
\hline Past or Current Use & $18(17.3 \%)$ & $86(82.7 \%)$ & $2.0(0.9,4.6)$ & 0.09 \\
\hline \multicolumn{5}{|l|}{ Laboratory Results } \\
\hline ESR, per $10 \mathrm{~mm} / \mathrm{hr}$ & $11(10-17)$ & $10(7-19)$ & $1.1(0.9,1.4)$ & 0.37 \\
\hline hsCRP level (mg/dL) & $0.2(0.1-0.4)$ & $0.1(0.1-0.3)$ & $0.8(0.4,1.9)$ & 0.69 \\
\hline Total cholesterol, per $10 \mathrm{mg} / \mathrm{dL}$ & $200.5(162-238)$ & $183(157-204)$ & $1.1(1.0,1.2)$ & 0.17 \\
\hline $\mathrm{HDL}$, per $10 \mathrm{mg} / \mathrm{dL}$ & $36(31-43)$ & $40(35-50)$ & $0.6(0.4,0.9)$ & 0.02 \\
\hline $\mathrm{LDL}$, per $10 \mathrm{mg} / \mathrm{dL}^{\mathrm{c}}$ & $104(76-132)$ & $101(87-129)$ & $1.0(0.9,1.2)$ & 0.46 \\
\hline Triglyceride level, per 100 mg/dL & $188(145-399)$ & $135(87-235)$ & $1.4(1.1,1.7)$ & 0.002 \\
\hline Fasting glucose, per $10 \mathrm{mg} / \mathrm{dL}^{\mathrm{c}}$ & $97(92-105)$ & $91(85-98)$ & $1.3(1.0,1.5)$ & 0.01 \\
\hline \multicolumn{5}{|l|}{ Weight and Fat Measurements } \\
\hline BMI $\left(\mathrm{kg} / \mathrm{m}^{2}\right)$ & $30(27-32)$ & $26(24-29)$ & $1.2(1.1,1.3)$ & $<0.0001$ \\
\hline Waist circumference (cm) & $104(95-107)$ & $92(84-98)$ & $1.1(1.0,1.1)$ & $<0.0001$ \\
\hline Percent body fat ${ }^{c}$ & $28(24-33)$ & $26(23-29)$ & $1.1(1.0,1.1)$ & 0.01 \\
\hline \multicolumn{5}{|l|}{ Physician Visual Assessment } \\
\hline Lipohypertrophy & $20(19.6 \%)$ & $82(80.4 \%)$ & $3.1(1.3,7.3)$ & 0.01 \\
\hline Lipoatrophy & $10(12.5 \%)$ & $01(87.5 \%)$ & $0.9(0.4,2.0)$ & 0.75 \\
\hline
\end{tabular}

a Categorical variables expressed as number (percentage) and continuous variables as median (interquartile range) other weight and body measurements were no included due to collinearity

${ }^{b}$ Variables FRS, family history of CAD, and all other ART medications were removed from the table due to lack of significance with FLD;

${ }^{c}$ All values are $n=211$, except LDL $(n=198)$, glucose $(n=210)$ and percent body fat $(n=212)$ due to missing values

BMI, body-mass index; $\mathrm{Cl}, 95 \%$ confidence interval; ESR, erythrocyte sedimentation rate; HAART, highly active antiretroviral therapy; HDL, high-density lipoprotein; hsCRP, high sensitivity C-reactive protein; LDL, low-density lipoprotein; NRTI, nucleoside/nucleotide reverse transcriptase inhibitor; OR, odds ratio

Table 3: Study Population Characteristics and Univariate Associations with Fatty Liver Disease among HIV-infected Men 
between adiponectin levels and CAD as detected by CAC scores among HIV-infected persons. To our knowledge, only two prior studies have evaluated measures of cardiovascular disease and adiponectin levels among HIV patients. The first study examined 56 HIV patients and found that a high FRS score was related to hypoadiponectinemia, but no cardiac imaging was performed [25]. The second study $(n=45)$ noted a relationship between low adiponectin levels and coronary flow reserve by echocardiography [56]. Our results are concurrent with these studies suggesting a potential association between hypoadiponectemia and CAD [26,59]. These data provide an additional potential pathogenic mechanism (i.e., low adiponectin levels) for the increased risk of cardiovascular diseases noted among HIV patients.

Low adiponectin levels in our study were also correlated with lipohypertrophy, increased BMI, elevated triglyceride levels, and hypertension, concurrent with prior reports $[25,51,54,56]$. Additionally, our study noted inverse correlations between adiponectin and duration of HIV infection, cumulative NRTI use, and didanosine. These HIVrelated factors have been associated with progressive lipodystrophy and cardiovascular disease, and our findings may provide insight into interrelated pathogenic mechanisms.

The importance of understanding the potential role of adiponectin in the pathogenesis of cardiovascular disease among HIV-infected persons may include the development of novel preventive and treatment strategies. For example, treatment with growth hormone-releasing hormone analogues has been associated with increased adiponectin levels and decreased Visceral Adipose Tissue (VAT) [57]. Studies have also evaluated the use of PPAR- $\gamma$ agonists, such as thiazolidinediones, for lipoatrophy since altered adipogenesis and PPAR- $\gamma$ signaling may reduce adiponectin levels. In small studies, rosiglitazone increased adiponectin levels, but had variable results on altering fat distribution [60,61]. Finally, in mice models, direct administration of adiponectin has been shown to ameliorate some of the adverse metabolic effects of PIs [50]. Understanding the potential benefit of increasing adiponectin levels, including on cardiovascular disease, should be a focus of future research studies.

In addition to treatment strategies, adiponectin levels could also represent an important marker for patients at higher risk of cardiovascular disease. Other markers of inflammation and kidney reserve have already been shown to correlate with future cardiovascular risk including hsCRP, D-dimer, and cystatin C levels [4,25,62-64]. Interestingly, inflammatory markers themselves (such as hsCRP) can down-regulate adiponectin production, potentially explaining some of the predictive capabilities of these markers [65]. Whether the detection of altered marker levels (e.g., low adiponectin levels) should result in intensified cardiovascular screening is currently unknown, and prospective studies are needed especially among HIV-infected persons who are at higher risk for these outcomes.

We examined factors associated with FLD and found that increased triglyceride levels and waist circumference were significantly associated, similar to other studies in both HIV-infected and HIV-uninfected persons. We did not find an association with adiponectin levels; this may be due to the small number of patients with FLD in our cohort, since the pathogenesis of CAD and FLD appears to overlap [66].

Our study had some limitations including the cross-sectional study design; hence we could not assess the temporality between low adiponectin levels and the development of CAD. Second, CAC is surrogate for $\mathrm{CAD}$ and we did not evaluate clinical cardiovascular events. However, CAC scores are increasingly utilized in this population for detection of CAD [67] and have been shown to predict future cardiovascular events [30]; hence, our study may have important clinical implications. Third, our study was solely among HIV-infected men, hence our results may not be applicable to women. Fourth, we did not differentiate adiponectin into low- and high-molecular forms, but rather examined total levels. Additionally, the diagnosis of lipodystrophy was by visual assessment and did not include DEXA scans. Finally, our results should be contextualized to the population studied; our cohort had a high prevalence ( $60 \%)$ of lipodystrophy, hence our results may not be applicable among other HIV-positive groups.

There were several strengths of our study. Our study is one of the first to examine the association of adiponectin and CAD, and had a more robust sample size compared to prior studies $[25,56]$. Additionally we examined several clinical, laboratory, and anthropometric/body measurements in addition to adiponectin levels with CAD. Finally, the findings from this study add to the existing literature on CAD and HIV-infected men; since HIV patients appear to be at higher risk for coronary events, early assessment of risk using novel markers and a better understanding of the pathogenesis may ultimately help increase the lifespan of patients.

In summary, low adiponectin levels are common among HIVinfected men and are correlated with the presence of lipohypertrophy. Hypoadiponectinemia had a marginal association with CAD, and may represent a novel marker and target for future treatment strategies among HIV-infected persons.

\section{References}

1. Palella FJ Jr, Baker RK, Moorman AC, Chmiel JS, Wood KC, et al. (2006) Mortality in the highly active antiretroviral therapy era: changing causes of death and disease in the HIV outpatient study. J Acquir Immune Defic Syndr 43: $27-34$

2. Antiretroviral Therapy Cohort Collaboration (2010) Causes of death in HIV-1 infected patients treated with antiretroviral therapy, 1996-2006: collaborative analysis of 13 HIV cohort studies. Clin Infect Dis 50: 1387-1396.

3. Tien PC, Choi Al, Zolopa AR, Benson C, Tracy R, et al. (2010) Inflammation and mortality in HIV-infected adults: analysis of the FRAM study cohort. J Acquir Immune Defic Syndr 55: 316-322.

4. Mangili A, Polak JF, Quach LA, Gerrior J, Wanke CA (2011) Markers of atherosclerosis and inflammation and mortality in patients with HIV infection. Atherosclerosis 214: 468-473.

5. Guaraldi G, Zona S, Alexopoulos N, Orlando G, Carli F, et al. (2009) Coronary aging in HIV-infected patients. Clin Infect Dis 49: 1756-1762.

6. Medina S, Wessman D, Krause D, Stepenosky J, Boswell G, et al. (2010) Coronary aging in HIV-infected patients. Clin Infect Dis 51: 990-993.

7. Hsue PY, Hunt PW, Schnell A, Kalapus SC, Hoh R, et al. (2009) Role of vira replication, antiretroviral therapy, and immunodeficiency in HIV-associated atherosclerosis. AIDS 23: 1059-1067.

8. Palella FJ Jr, Phair JP (2011) Cardiovascular disease in HIV infection. Curr Opin HIV AIDS 6: 266-271.

9. Fichtenbaum CJ (2009) Metabolic abnormalities associated with HIV infection and antiretroviral therapy. Curr Infect Dis Rep 11: 84-92.

10. Phillips AN, Carr A, Neuhaus J, Visnegarwala F, Prineas R, et al. (2008) Interruption of antiretroviral therapy and risk of cardiovascular disease in persons with HIV-1 infection: exploratory analyses from the SMART trial. Antivir Ther 13: 177-187.

11. DAD Study Group, Friis-Møller N, Reiss P, Sabin CA, Weber R, et al. (2007) Class of antiretroviral drugs and the risk of myocardial infarction. N Engl J Med 356: 1723-1735.

12. Lang S, Mary-Krause M, Cotte L, Gilquin J, Partisani M et al. (2010) Impact of individual antiretroviral drugs on the risk of myocardial infarction in human immunodeficiency virus-infected patients: a case-control study nested within 
Citation: Norton L, Brodine SK, Medina S, Lemus H, Ricchiuti V, et al. (2013) Adiponectin Levels and Coronary Artery Disease among HIV-Infected Men. J AIDS Clin Res 4: 208. doi:10.4172/2155-6113.1000208

Page 8 of 9

the French Hospital Database on HIV ANRS cohort CO4. Arch Intern Med 170 1228-1238.

13. Worm SW, Sabin C, Weber R, Reiss P, El-Sadr W, et al. (2010) Risk of myocardial infarction in patients with HIV infection exposed to specific individual antiretroviral drugs from the 3 major drug classes: the data collection on adverse events of anti-HIV drugs (DAD) study. J Infect Dis 201: 318-330.

14. Plutzky J (2001) Inflammatory pathways in atherosclerosis and acute coronary syndromes. Am J Cardiol 88: 10K-15K.

15. Hansson GK (2005) Inflammation, atherosclerosis, and coronary artery disease. N Engl J Med 352: 1685-1695.

16. Danesh J, Wheeler JG, Hirschfield GM, Eda S, Eiriksdottir G, et al. (2004) C-reactive protein and other circulating markers of inflammation in the prediction of coronary heart disease. N Engl J Med 350: 1387-1397.

17. Sweeney LL, Brennan AM, Mantzoros CS (2007) The role of adipokines in relation to HIV lipodystrophy. AIDS 21: 895-904.

18. Okamoto Y, Kihara S, Funahashi T, Matsuzawa Y, Libby P (2006) Adiponectin: a key adipocytokine in metabolic syndrome. Clin Sci (Lond) 110: 267-278.

19. Kadowaki T, Yamauchi T, Kubota N, Hara K, Ueki K, et al. (2006) Adiponectin and adiponectin receptors in insulin resistance, diabetes, and the metabolic syndrome. J Clin Invest 116: 1784-1792

20. Hopkins TA, Ouchi N, Shibata R, Walsh K (2007) Adiponectin actions in the cardiovascular system. Cardiovasc Res 74: 11-18.

21. Goldstein BJ, Scalia R (2004) Adiponectin: A novel adipokine linking adipocytes and vascular function. J Clin Endocrinol Metab 89: 2563-2568.

22. Ouchi N, Kihara S, Arita Y, Maeda K, Kuriyama H, et al. (1999) Novel modulator for endothelial adhesion molecules: adipocyte-derived plasma protein adiponectin. Circulation 100: 2473-2476.

23. Laughlin GA, Barrett-Connor E, May S, Langenberg C (2007) Association of adiponectin with coronary heart disease and mortality: the Rancho Bernardo study. Am J Epidemiol 165: 164-174

24. Okamoto Y (2011) Adiponectin provides cardiovascular protection in metabolic syndrome. Cardiol Res Pract 2011: 313179

25. Falasca K, Ucciferri C, Mancino P, Di lorio A, Vignale F, et al. (2010) Cystatin $C$, adipokines and cardiovascular risk in HIV infected patients. Curr HIV Res 8: 405-410.

26. Hadigan C, Meigs JB, Wilson PW, D'Agostino RB, Davis B, et al. (2003) Prediction of coronary heart disease risk in HIV-infected patients with fat redistribution. Clin Infect Dis 36: 909-916.

27. Tong Q, Sankalé JL, Hadigan CM, Tan G, Rosenberg ES, et al. (2003) Regulation of adiponectin in human immunodeficiency virus-infected patients relationship to body composition and metabolic indices. J Clin Endocrino Metab 88: 1559-1564.

28. Vigouroux C, Maachi M, Nguyên TH, Coussieu C, Gharakhanian S, et al. (2003) Serum adipocytokines are related to lipodystrophy and metabolic disorders in HIV-infected men under antiretroviral therapy. AIDS 17: 1503-1511.

29. Jan V, Cervera P, Maachi M, Baudrimont M, Kim M, et al. (2004) Altered fat differentiation and adipocytokine expression are inter-related and linked to morphological changes and insulin resistance in HIV-1-infected lipodystrophic patients. Antivir Ther 9: 555-564.

30. Pletcher MJ, Tice JA, Pignone M, Browner WS (2004) Using the coronary artery calcium score to predict coronary heart disease events: a systematic review and meta-analysis. Arch Intern Med 164: 1285-1292.

31. Crum-Cianflone N, Krause D, Wessman D, Medina S, Stepenosky J, et al. (2011) Fatty liver disease is associated with underlying cardiovascular disease in HIV-infected persons( $\left.{ }^{*}\right)$. HIV Med 12: 463-471.

32. Nishizawa H, Shimomura I, Kishida K, Maeda N, Kuriyama H, et al. (2002) Androgens decrease plasma adiponectin, an insulin-sensitizing adipocytederived protein. Diabetes 51: 2734-2741.

33. Panel on Antiretroviral Guidelines for Adults and Adolescents (2011) Guidelines for the use of antiretroviral agents in HIV-1-infected adults and adolescents.

34. Farrell GC, Larter CZ (2006) Nonalcoholic fatty liver disease: from steatosis to cirrhosis. Hepatology 43: S99-S112.

35. National Cholesterol Education Program (2002) Third report of the expert panel on detection, evaluation, and treatment of high blood cholesterol in adults. Circulation 106: 3143-3421.

36. Durnin JV, Womersley J (1974) Body fat assessed from total body density and its estimation from skinfold thickness: measurements on 481 men and women aged from 16 to 72 years. Br J Nutr 32: 77-97.

37. Pierson RN Jr, Wang J, Heymsfield SB, Russell-Aulet M, Mazariegos M, et al. (1991) Measuring body fat: calibrating the rulers. Intermethod comparisons in 389 normal Caucasian subjects. Am J Physiol 261: E103-108.

38. Mayo Clinic (1981) Mayo Clinic Diet Manual: A Handbook of Dietary Practices ( $5^{\text {th }}$ Edn.), WB Saunders Company, Philadelphia.

39. Lichtenstein KA, Ward DJ, Moorman AC, Delaney KM, Young B, et al. (2001) Clinical assessment of HIV-associated lipodystrophy in an ambulatory population. AIDS 15: 1389-1398.

40. Kumada M, Kihara S, Sumitsuji S, Kawamoto T, Matsumoto S, et al. (2003) Association of hypoadiponectinemia with coronary artery disease in men. Arterioscler Thromb Vasc Biol 23: 85-89.

41. Im JA, Kim SH, Lee JW, Shim JY, Lee HR, et al. (2006) Association between hypoadiponectinemia and cardiovascular risk factors in nonobese healthy adults. Metabolism 55: 1546-1550.

42. Agatston AS, Janowitz WR, Hildner FJ, Zusmer NR, Viamonte M Jr, et al. (1990) Quantification of coronary artery calcium using ultrafast computed tomography. J Am Coll Cardiol 15: 827-832.

43. Jacobs JE, Birnbaum BA, Shapiro MA, Langlotz CP, Slosman F, et al. (1998) Diagnostic criteria for fatty infiltration of the liver on contrast-enhanced helical CT. AJR Am J Roentgenol 171: 659-664.

44. Ricci C, Longo R, Gioulis E, Bosco M, Pollesello P, et al. (1997) Noninvasive in vivo quantitative assessment of fat content in human liver. J Hepatol 27 108-113.

45. Amorosa V, Synnestvedt M, Gross R, Friedman H, MacGregor RR, et al. (2005) A tale of 2 epidemics: the intersection between obesity and HIV infection in Philadelphia. J Acquir Immune Defic Syndr 39: 557-561.

46. Crum-Cianflone N, Roediger MP, Eberly L, Headd M, Marconi V, et al. (2010) Increasing rates of obesity among HIV-infected persons during the HIV epidemic. PLoS One 5: e10106.

47. Dube' MP, Zackin R, Tebas P, Roubenoff R, Mulligan K, et al. (2002) Prospective study of regional body composition in antiretroviral-naïve subjects randomized to receive zidovudine and lamivudine or didanosine and stavudine combined with nelfinavir, efavirenz, or both: A5005s, a study of ACTG 384. Antivir The 7: L27.

48. Jones SP, Waitt C Sutton R, Back DJ, Pirmohamed M (2008) Effect of atazanavir and ritonavir on the differentiation and adipokine secretion of human subcutaneous and omental preadipocytes. AIDS 22: 1293-1298.

49. Vernochet C, Azoulay S, Duval D, Guedj R, Cottrez F, et al. (2005) Human immunodeficiency virus protease inhibitors accumulate into cultured human adipocytes and alter expression of adipocytokines. J Biol Chem 280: 22382243.

50. Xu A, Yin S, Wong L, Chan KW, Lam KS (2004) Adiponectin ameliorates dyslipidemia induced by the human immunodeficiency virus protease inhibitor ritonavir in mice. Endocrinology 145: 487-494.

51. Addy C, Gavrila A, Tsiodras S, Brodovicz K, Karchmer A, et al. (2003) Hypoadiponectinemia is associated with insulin resistance, hypertriglyceridemia and fat redistribution in human immunodeficiency virus-infected patients treated with highly active antiretroviral therapy. J Clin Endocrinol Metab 88: 627-636.

52. Calmy A, Hirschel B, Cooper DA, Carr A (2007) Clinical update: adverse effects of antiretroviral therapy. Lancet 370: 12-14.

53. Li FY, Cheng KK, Lam KS, Vanhoutte PM, Xu A (2011) Cross-talk between adipose tissue and vasculature: role of adiponectin. Acta Physiol (Oxf) 203: 167-180.

54. Luo L, Zhang L, Tao M, Qiu Z, Xie J, et al. (2009) Adiponectin and leptin levels in Chinese patients with HIV-related lipodystrophy: a 30-month prospective study. AIDS Res Hum Retroviruses 25: 1265-1272.

55. Hui X, Lam KS, Vanhoutte PM, Xu A (2012) Adiponectin and cardiovascula health: an update. Br J Pharmacol 165: 574-590.

56. Bezante GP, Briatore L, Rollando D, Maggi D, Setti M, et al. (2009) 
Citation: Norton L, Brodine SK, Medina S, Lemus H, Ricchiuti V, et al. (2013) Adiponectin Levels and Coronary Artery Disease among HIV-Infected Men. J AIDS Clin Res 4: 208. doi:10.4172/2155-6113.1000208

Page 9 of 9

Hypoadiponectinemia in lipodystrophic HIV individuals: a metabolic marker of subclinical cardiac damage. Nutr Metab Cardiovasc Dis 19: 277-282.

57. Stanley TL, Falutz J, Mamputu JC, Soulban G, Potvin D, et al. (2011) Effects of tesamorelin on inflammatory markers in HIV patients with excess abdominal fat: relationship with visceral adipose reduction. AIDS 25: 1281-1288.

58. Wolk R, Berger P, Lennon RJ, Brilakis ES, Davison DE, et al. (2007) Association between plasma adiponectin levels and unstable coronary syndromes. Eur Heart J 28: 292-298.

59. Pischon T, Girman CJ, Hotamisligil GS, Rifai N, Hu FB, et al. (2004) Plasma adiponectin levels and risk of myocardial infarction in men. JAMA 291: 17301737.

60. Hadigan C, Yawetz S, Thomas A, Havers F, Sax PE, et al. (2004) Metabolic effects of rosiglitazone in HIV lipodystrophy: a randomized, controlled trial. Ann Intern Med 140: 786-794.

61. Blümer RM, van der Valk M, Ackermans M, Endert E, Serlie MJ, et al. (2009) A rosiglitazone-induced increase in adiponectin does not improve glucose metabolism in HIV-infected patients with overt lipoatrophy. Am J Physiol Endocrinol Metab 297: E1097-1104.
62. Baker JV, Duprez D (2010) Biomarkers and HIV-associated cardiovascular disease. Curr Opin HIV AIDS 5: 511-516.

63. Ford ES, Greenwald JH, Richterman AG, Rupert A, Dutcher L, et al. (2010) Traditional risk factors and D-dimer predict incident cardiovascular disease events in chronic HIV infection. AIDS 24: 1509-1517.

64. Pisto P, Ukkola O, Santaniemi M, Kesäniemi YA (2011) Plasma adiponectin-an independent indicator of liver fat accumulation. Metabolism 60: 15151520

65. Yuan G, Chen X, Ma Q, Qiao J, Li R, et al. (2007) C-reactive protein inhibits adiponectin gene expression and secretion in 3T3-L1 adipocytes. J Endocrinol 194: $275-281$.

66. Nseir W, Shalata A, Marmor A, Assy N (2011) Mechanisms linking nonalcoholic fatty liver disease with coronary artery disease. Dig Dis Sci 56: 3439-3449.

67. Mangili A, Gerrior J, Tang AM, O'Leary DH, Polak JK, et al. (2006) Risk of cardiovascular disease in a cohort of HIV-infected adults: a study using carotid intima-media thickness and coronary artery calcium score. Clin Infect Dis 43 : 1482-1489. 